\title{
Adding Injectable Chitosan Poloxamer to Platelet Rich Plasma (PRP) Has no Beneficial Effect in Knee OA. A prospective Experimental Study in Guinea Pigs
}

\author{
T. Bansal ${ }^{1}$, S. Patel ${ }^{1}$, D. K. Chouhan ${ }^{1}$, A. Bhatia' ${ }^{2}$ B. Medhi ${ }^{3}$, N. Kakkar ${ }^{4}$, M. Singh Dhillon ${ }^{1}$ \\ 1 Department of Orthopaedics, Post Graduate Institute of Medical Education and Research (PGIMER), \\ Chandigarh, India \\ 2 Department of Experimental Medicine and Biotechnology, Post Graduate Institute of Medical Education \\ and Research (PGIMER), Chandigarh, India \\ 3 Department of Pharmacology, Post Graduate Institute of Medical Education and Research (PGIMER), \\ Chandigarh, India \\ 4 Department of Pathology, Post Graduate Institute of Medical Education and Research (PGIMER), Chandigarh, \\ India
}

\section{CORRESPONDING AUTHOR:}

Sandeep Patel

Department of Orthopaedics

Post Graduate Institute of Medical

Education and Research (PGIMER)

Chandigarh, 160012

India

E-mail: sandeepdrpatelortho@gmail.com

DOI:

10.32098/mltj.04.2020.04

LEVEL OF EVIDENCE: 5

\begin{abstract}
SUMMARY
Background. Short term symptomatic relief with single dose PRP in early knee OA has been established. We studied the effect of Chitosan as an additive to PRP, in an attempt to improve and prolong the effects.

Methods. 42 Dunkin Hartley guinea pigs were divided into 4 groups of 10 each; 2 animals were used for preparation of allogeneic PRP. Disease control group (DC) had 10 animals where no intervention was done in either knee. Groups G1, G2 and G3 (10 animals each) were given single intra-articular injections of PRP, Chitosan gel alone and Chitosan + PRP in one knee respectively. Isotonic saline injection was given in the contralateral knees in all 3 groups to act as control. Five animals from each group (subgroup DC.3, G1.3, G2.3 and G3.3) were euthanized at three months and the remaining five (subgroup DC.6, G1.6, G2.6 and G3.6) at six months post intervention. Upon euthanasia, knee joint synovial fluid was taken for cartilage oligomeric protein (COMP) estimation by ELISA, and histologic assessment of articular cartilage and synovium was done using Mankin score.

Results. The mean synovitis scores was significantly lower in both the PRP and Chitosan PRP group compared to disease control and Chitosan gel alone groups at 3 months $(\mathrm{p}<0.05)$ signifying effectiveness of PRP for synovitis; at 6 months there was no difference between the 3 intervention groups and disease control group, implying that the effects of PRP wear off. There was no significant difference between Chitosan PRP and PRP groups at 3 or 6 months in terms of mean total articular cartilage score and COMP levels. The above findings imply that Chitosan addition did not improve or prolong the effects of PRP.

Conclusions. Addition of chitosan poloxamer gel did not offer any additional advantage in prolonging efficacy of PRP in a Guinea pig Knee OA model. Nevertheless, the efficacy of single dose PRP, with or without Chitosan, has been again demonstrated in the short term, due to decreased synovitis scores.
\end{abstract}

\section{KEY WORDS}

Chitosan; poloxamer gel; platelet rich plasma; guinea pigs; animal study; COMP. 


\section{INTRODUCTION}

In the $21^{\text {st }}$ century, Platelet Rich Plasma (PRP) has emerged as a promising treatment option for early osteoarthritis of knee. The positive effects of PRP have been noted by multiple authors $(1,2,3)$; however, the downside is that the effect tends to deteriorate after periods of time varying from 6 to 12 months (3).

PRP has been combined with many different biomaterials like gelatin hydrogels, Chitosan, PLGA (poly lactic-co-glycolic acid) mesh and $\beta$-tricalcium phosphate scaffolds for prolonged and sustained release of growth factors, with varying degrees of success. The combination of PRP with these different biomaterials has been demonstrated to be significantly better than PRP alone in various in vitro and in vivo studies for various applications like wound healing, healing of bone defects and for osteoarthritis knee (4-9).

Chitosan has been shown to improve some platelet properties. It increases platelet aggregation and adhesion and increases the release of growth factors $(10,11)$. Chitosan scaffolds activated with PRP are safe and nontoxic (12) and have displayed controlled and sustained release of growth factors $(12,13)$. Many in vivo studies have also demonstrated the improved efficacy of Chitosan and PRP combination for wound healing and healing of bone defects (14, 15). Chitosan and PRP combination scaffolds have been successfully used in focal cartilage defect models in rabbit where better quality of cartilage tissue with PRP+ Chitosan implants has been noted compared to controls $(16,17)$. Dwivedi et al. (17) had used injectable Chitosan PRP which solidifies in the defect to behave as a scaffold. Injectable Chitosan gels have been used in healing of abdominal defects and in preventing peritoneal adhesions $(18,19)$. In the present study we explored the potential of injectable Chitosan and injectable Chitosan + PRP combination for use in Osteoarthritis model. Considering the positive effects in cartilage healing demonstrated by Chitosan + PRP scaffolds in focal defects, we hypothesized that this combination may enhance and prolong the effect of PRP in cartilage healing in osteoarthritis, and prospectively designed a study in a Guinea Pig model of OA. A comparative experimental study was done to determine whether Chitosan poloxamer + PRP is better than PRP alone for osteoarthritis in guinea pig knee, based on histopathological analysis of synovium and cartilage.

\section{MATERIAL AND METHODS}

\section{Selection of animals for study and grouping}

Forty-two male Dunkin-Hartley guinea pigs, 5 months of age and weighing approximately 600 to 800 grams were chosen for the study after institutional animal ethics committee clearance (IAEC number 547). The Dunkin Hartley guinea pigs represent a naturally occurring model of osteoarthritis, where the progression of osteoarthritis is affected by increasing age and weight gain (20). 2 animals were used for preparation of allogeneic PRP (Donor Group). The remaining 40 animals constituted the experimental group. The study animals were divided into 4 groups randomly as described and shown in figure 1. Each knee of the 40 animals was considered a separate entity and therefore a total of 80 knees were available for the study. Therapeutic intervention was done on one knee (randomly selected) and the contra-lateral knee was taken as control for each animal in study all groups.

Group DC: Disease control group - This group had 10 animals, which were further divided into subgroups of 5 animals each, which were sacrificed at 3 and 6 months (DC.3 and DC.6) respectively. Both knees received no intervention, giving a total of 20 knees.

Group G1: PRP Injection group - This group contained 10 animals. These were further divided into subgroups containing 5 animals each, which were sacrificed at 3 and 6 months (G1.3 and G1.6) respectively. Single PRP injection was given in the intervention knee and same amount of saline was injected in the control knee.

Group G2: Chitosan Injection group - This group contained 10 animals. These were further sub divided into groups containing 5 animals each, which were sacrificed at 3 and 6 months (G2.3 and G2.6) respectively. Single injection of Chitosan gel was given in the intervention knee and same amount of saline was injected in the control knee.

Group G3: Chitosan-PRP combination injection group This group contained 10 animals. These were further sub divided into groups containing 5 animals each which were sacrificed at 3 and 6 months (G3.3 and G3.6) respectively. A single injection of Chitosan poloxamer PRP gel was given in the intervention knee and same amount of saline was injected in the control knee.

The animals were housed according to subgroups in groups of 5 each in open rectangular metal floor pen of $45 \times 90 \mathrm{~cm}$ size. The bedding was made with straw with free access to food and water and maintained on a $12 \mathrm{hr}$ light/12 hr dark cycle $(8,20)$. All standard ethical guidelines as stated by this journal were followed (21).

The mean synovitis scores, the mean total articular scores and mean COMP concentrations of control arm of various groups (DC, G1, G2 and G3) at 3 months and 6 months were calculated (table I). The control arms were comparable to each other ( $\mathrm{p}$ value $>0.05$ for all comparisons) at 3 months and 6 months (table II), implying that Osteoarthritis progression in all groups was similar and comparable. 


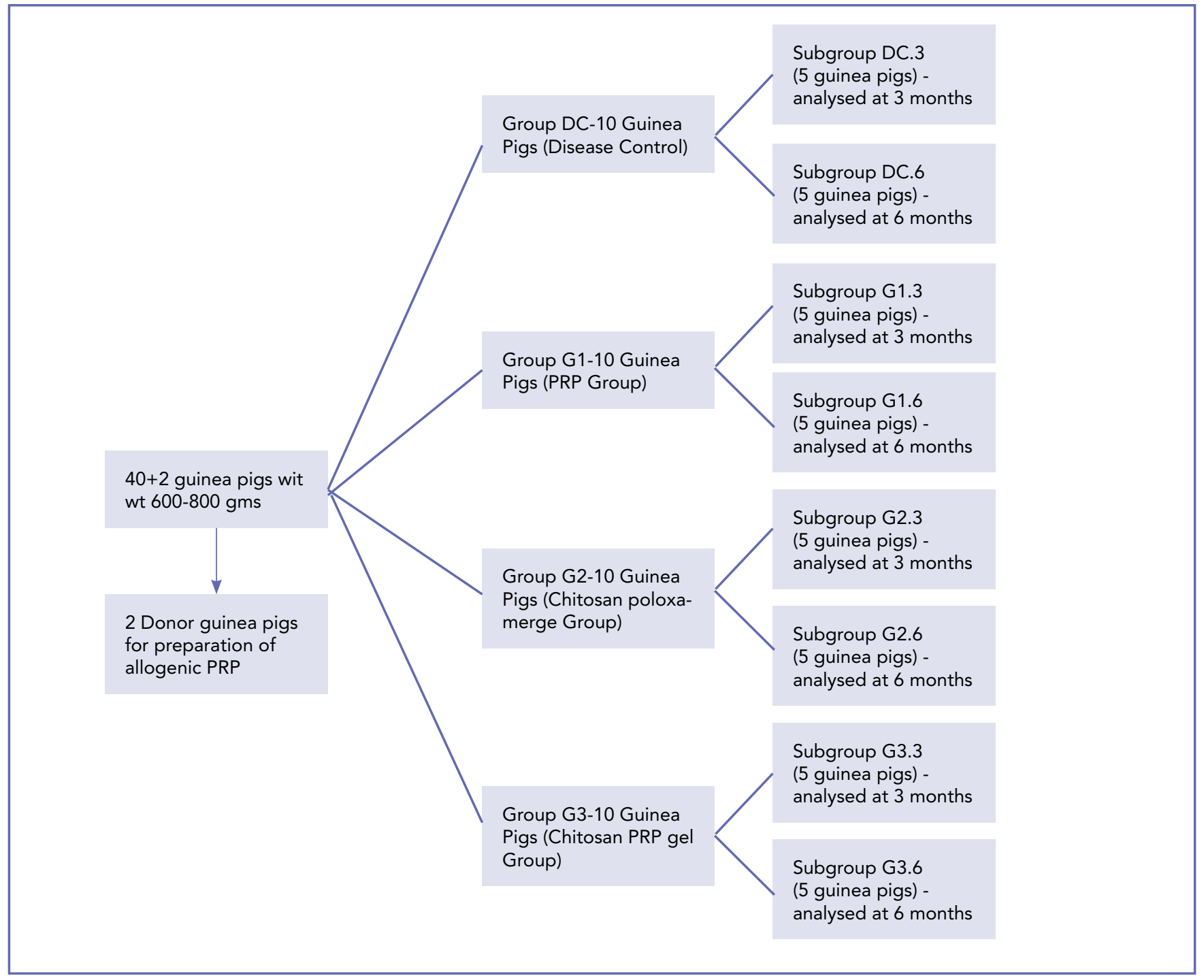

Figure 1. Pictorial representation of the distribution of animal among various groups. Group DC-Disease control group, Group 1 - PRP group, Group 2 - Chitosan only group, Group 3 - Chitosan PRP group.

Table I. Mean Synovitis score, Mean Total Articular scores and mean COMP scores of CONTROL Arm of all 8 subgroups.

\begin{tabular}{lllll}
\hline S1 No & Group & $\begin{array}{l}\text { Synovitis score } \\
\text { (Mean } \pm \text { Std deviation) }\end{array}$ & $\begin{array}{l}\text { Total Articular Score } \\
(\text { Mean } \pm \text { Std deviation) }\end{array}$ & Mean synovial COMP Levels \\
\hline 1 & DC. 3 & $3.40 \pm .894$ & $8.60 \pm 3.209$ & $7990.00 \pm 2403.258$ \\
\hline 2 & G1.3 & $3.00 \pm 2.828$ & $8.80 \pm 1.924$ & $6818.00 \pm 2258.942$ \\
\hline 3 & G2.3 & $3.80 \pm 1.924$ & $9.00 \pm 2.646$ & $6898.60 \pm 568.507$ \\
\hline 4 & G3.3 & $3.20 \pm 1.304$ & $9.40 \pm 2.881$ & $5676.60 \pm 1117.429$ \\
\hline 5 & DC.6 & $6.20 \pm 1.643$ & $12.60 \pm 1.140$ & $10084.60 \pm 1365.321$ \\
\hline 7 & G1.6 & $5.60 \pm 1.817$ & $13.00 \pm 3.000$ & $14976.00 \pm 1585.257$ \\
\hline 8 & G2.6 & $5.80 \pm 1.643$ & $11.20 \pm 1.643$ & $12622.20 \pm 1356.354$ \\
\hline
\end{tabular}


Table II. Table showing p values for comparison among CONTROL arm of various groups for mean synovial score, mean articular score and mean synovial COMP levels.

\begin{tabular}{lllll}
\hline $\begin{array}{l}\text { Subgroup 1 } \\
\text { for comparison }\end{array}$ & $\begin{array}{l}\text { Subgroup 2 } \\
\text { for comparison }\end{array}$ & $\begin{array}{l}\text { P value for comparison } \\
\text { of mean synovial scores }\end{array}$ & $\begin{array}{l}\text { P value for comparison } \\
\text { of mean articular cartilage scores }\end{array}$ & $\begin{array}{l}\text { P value for mean } \\
\text { synovial COMP levels }\end{array}$ \\
\hline DC.3 & G1.3 & 1.000 & 1.000 & .997 \\
\hline DC.3 & G2.3 & 1.000 & 1.000 & .998 \\
\hline DC.3 & G3.3 & 1.000 & .997 & .943 \\
\hline G1.3 & G2.3 & .989 & 1.000 & 1.000 \\
\hline G1.3 & G3.3 & 1.000 & .999 & .998 \\
\hline G2.3 & G3.3 & .997 & 1.000 & .997 \\
\hline DC.6 & G1.6 & .997 & 1.000 & .802 \\
\hline DC.6 & G2.6 & 1.000 & .954 & .991 \\
\hline DC.6 & G3.6 & 1.000 & .996 & .904 \\
\hline G1.6 & G2.6 & 1.000 & .878 & .983 \\
\hline G2.6 & G3.6 & .987 & .976 & 1.000 \\
\hline
\end{tabular}

\section{Preparation of platelet rich plasma (PRP)}

We used allogeneic blood obtained from donor animals via cardiac puncture for preparation of PRP. Double spin technique was used for preparation of PRP, as it has been shown to yield higher concentration of platelets compared to single spin technique (22). Blood was collected in vials containing acid citrate dextrose (ACD) containing $0.48 \%$ $\mathrm{w} / \mathrm{v}$ citric acid, $1.32 \% \mathrm{w} / \mathrm{v}$ sodium citrate and $1.47 \%$ dextrose. $1 \mathrm{ml}$ of this anticoagulant was added to each $10 \mathrm{ml}$ vial. Drawn blood was analyzed for platelet count; then it was subjected to double centrifugation. The centrifugation protocol used was 20 minutes of soft spin at $800 \mathrm{~g}$ which separated the blood into a red cell layer and a buffy coat. The buffy coat was pipetted out and subjected to hard spin for 15 minutes at $2200 \mathrm{~g}$. This yielded platelet poor plasma overlying platelet pellet at the bottom of the centrifugation tube. Two-third of the platelet poor plasma was removed, and the rest was evenly dissolved with the platelet pellet to produce a homogenous solution. While preparing PRP for Chitosan PRP group, PRP was concentrated doubly (see reason below) obtained by removing five-sixth of the platelet poor plasma and dissolving the platelet pallet with the rest of plasma $(20,23) .15 \mathrm{ml}$ whole blood produced approximately $3 \mathrm{ml}$ of allogenic PRP. The obtained PRP was subjected to platelet count for quality control, done manually using a Nuebaeur chamber. The normal guinea pig platelet count reference range is $3.4^{-10} \times 10^{5} / \mathrm{mm}(24)$; the PRP prepared had platelet counts at least 2 to 3 times the baseline (25).

The allogeneic PRP thus produced was injected into the joint immediately after preparation.

\section{Activation of PRP}

The PRP was activated by adding $\mathrm{CaCl}_{2}$ prior to injection; 1 part $0.025 \mathrm{M} \mathrm{CaCl}_{2}$ was added to 4 parts of allogenic PRP. Fresh $\mathrm{CaCl}_{2}$ solution was prepared for each injection by dissolving $27.78 \mathrm{mg}$ of anhydrous $\mathrm{CaCl}_{2}$ in $100 \mathrm{ml}$ distilled water. This solution was mixed in the ratio 1:4 with PRP injection in group 1. No activation of PRP was done for Group 3 (Chitosan PRP group) as Chitosan has been shown to be an activator of PRP (10).

\section{Preparation of Injectable Chitosan poloxamer gel}

The Chitosan solution $(1.33 \% \mathrm{w} / \mathrm{w})$ was prepared by mixing Chitosan (Sigma Aldrich ${ }^{\mathrm{TM}}$ medium molecular weight) in acetic acid solution $(0.25 \% \mathrm{w} / \mathrm{v})$ with continuous stirring until complete dissolution. The required amount of poloxamer 188 (Sigma Aldrich ${ }^{\mathrm{TM}}$ ) was added to Chitosan solution to obtain a $26.6 \%(\mathrm{w} / \mathrm{v})$ solution and the partially dissolved solution was kept in the refrigerator (at around $4{ }^{\circ} \mathrm{C}$ ) until the entire polymer completely dissolved (approximately 24 h). The Chitosan poloxamer solution so obtained was sterilized by autoclaving at $121{ }^{\circ} \mathrm{C}(250 \mathrm{~F})$ for 20 minutes at 15 psi (26). The ratio of Chitosan and poloxamer in the final preparation were $1 \% \mathrm{w} / \mathrm{w}$ and $20 \% \mathrm{w} / \mathrm{v}$ respectively. The rheological properties and gelling of Chitosan poloxamer solution before and after sterilization were checked. The force shear graphs obtained before sterilization and after sterilization were linear in nature and represented Newtonian type of fluid. There was no change in the rheological properties of the preparation after sterilization. The transition temperature of solution to gel was 30 to $32^{\circ} \mathrm{C}$. 


\section{Preparation of Injectable PRP-Chitosan poloxamer gel combination}

The guinea pig knee is small and accommodates around 100 microliters of fluid. As we had to combine PRP with Chitosan solution, the volume of both injected would have resulted in lesser volume/dose of PRP. In order to counter this and ensure adequate Platelet numbers in the PRP, we used a double concentrate PRP for group G3. The initial steps of PRP preparation were same which included double spin technique. Double concentrate PRP was then obtained by removing five-sixth of the platelet poor plasma and dissolving the platelet pellet in the rest of plasma. The Chitosan PRP group had half the volume of PRP in contrast to PRP alone group. The sterilized Chitosan solution was added to the double concentrate PRP solution under sterile conditions in the ratio 3:1 (Chitosan: PRP). To counter the dilution we used a double concentrate PRP in the chitosan PRP group and did not use an activator in the chitosan PRP group as chitosan is itself an activator of PRP. The preparation was kept at room temperature before injecting.

\section{Joint injections}

PRP and other preparations were instilled in the knee joint chosen for therapy through the inferior patellar tendon with a 26-gauge needle and syringe (100 microliters); the same amount of normal saline was injected in the other knee of the animal $(23,27)$. During the procedure the guinea pigs were anesthetized with a mixture of Xylazine $(5 \mathrm{mg} / \mathrm{kg})+$ ketamine $(50 \mathrm{mg} / \mathrm{kg})+$ acepromazine $(1 \mathrm{mg} / \mathrm{kg})$ given at a dose of $0.1 \mathrm{ml} /$ $\mathrm{kg}$ i. $\mathrm{m}$ injection and monitored. The procedure was conducted using sterile conditions. After the procedure the animals were monitored until they recovered from the anesthesia.

\section{Collection of synovial fluid}

We collected synovial fluid samples from each knee to perform Cartilage Oligomeric Protein (COMP) (a marker of cartilage degradation) level measurements. The synovial fluid samples were collected by the technique described previously after euthanasia of animals $(23,27)$. Through the inferior patellar tendon 200 microlitres isotonic saline was injected intra articularily. The limb was subjected to 10 cycles of flexion and extension of knee joint to evenly distribute the injected fluid. With the same approach and a 26 gauge needle the knee was aspirated.100 micro liters of sample was collected from each knee and the collected fluid was then subjected to centrifugation at $2000 \mathrm{rpm}$ for 10 minutes to remove cell debris and the supernatant was collected and stored at $-20^{\circ} \mathrm{C}$ for analysis. The samples were collected at the time of sacrificing the animal, before the joints were processed for histopathology.

\section{Analysis of synovial fluid for COMP levels}

The collected synovial fluid aliquots from each knee were thawed and subjected to quantitative COMP levels analysis by Enzyme Linked Immunosorbent Assay (ELISA) by a blinded observer. The ELISA kits (MyBiosource Elisa kits) use double sandwich ELISA technique and the kit had a sensitivity of $0.05 \mathrm{ng} / \mathrm{ml}$. The kit was stored at $-20{ }^{\circ} \mathrm{C}$ until usage. The samples were analyzed following the steps as given in the user manual provided by the manufacturer.

\section{Collection and analysis of synovial tissue and joint cartilage}

Under xylazine and ketamine anesthesia, euthanasia was performed on the animal by intra peritoneal injection of pentobarbital $(100 \mathrm{mg} / \mathrm{kg})$. The animal was observed for the loss of respiratory drive and loss of vital parameters before starting the tissue harvest $(23,28)$. The harvested tissue was then fixed in $10 \%$ formaldehyde and subjected to histological analysis.

The harvested tissues were fixed in 10\% formalin and both knee joints for each animal were assessed. The samples were processed using saturated solution of EDTA in 0.1 $\mathrm{M}$ phospahate buffer. A $\mathrm{pH}$ of approximately 7.6-7.8 was maintained. The solution was replaced once every week until calcium precipitated was no longer detected. This took approximately 8-12 weeks. Further, paraffin wax blocks were made $(2,29)$. For articular cartilage staining toluidine blue stain was used and scoring was done using semi quantitative Mankin score which has been validated by the Osteoarthritis Research Society International (OARSI) histopathology initiative in guinea pigs (29). The score has 5 components which included articular cartilage score (0-8), proteoglycan content (0-6), cellularity (0-3), tidemark (0-1) and osteophytes (0-3) with a maximum score of 21 and a minimum score of 0 (29). Synovial tissue was stained with heamatoxylin and eosin and was analyzed according to a semi-quantitative score given by Pelletier et al. and validated and recommended by the OARSI histopathology initiative (29). The scoring has 3 components which include synovial hyperplasia (0-2), villous hyperplasia (0-3) and degree of cellular infiltration (0 or 5). The minimum score was 0 and maximum was 10 (29). An independent blinded observer did all scorings.

\section{STATISTICS}

The collected data was subjected to statistical analysis using SPSS v20 software. The descriptive statistics for all 8 subgroups (e.g. Mean, standard deviation etc.) were calculated for weight, platelet counts in whole blood and PRP, synovitis scores, articular cartilage scores and COMP levels. 
The data was confirmed to be normally distributed. Weight gain, total synovial scores, total articular scores and COMP levels were compared among various groups using one way analysis of variance and post hoc Tukey HSD tests. A p value of $<0.05$ was considered significant.

\section{RESULTS}

The mean initial weights at the start of experiment in all subgroups, the mean weight at sacrifice at 3 months and 6 months, and the mean weight gain of animal subgroups sacrificed at 3 months and 6 months were all comparable (figure 2), with no significant difference among the groups. The $\mathrm{p}$ values for these comparisons are listed in table III. These animals were thus comparable in terms of osteoarthritis (29).

The baseline platelet counts in source blood used for the PRP injection of group G1 and group G3 were 585,000/ $\mu \mathrm{l}$ and $640,000 / \mu \mathrm{l}$. The platelet counts in PRP injection prepared for group G1 was 1,875,000/ $\mu \mathrm{l}$ and PRP injec-

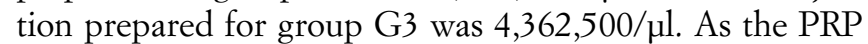
used for injection in group G3 was mixed with Chitosan poloxamer preparation in the ratio 1:3, the platelet concentration in final preparation was $1090625 / \mu l$. The final PRP concentration in group $\mathrm{G} 1$ after activation with $\mathrm{CaCl}^{2}$ was $1,500,000 / \mu \mathrm{l}$.

The mean synovitis scores for intervention arm of all groups are represented in figure 3 . At 3 months, the scores for both

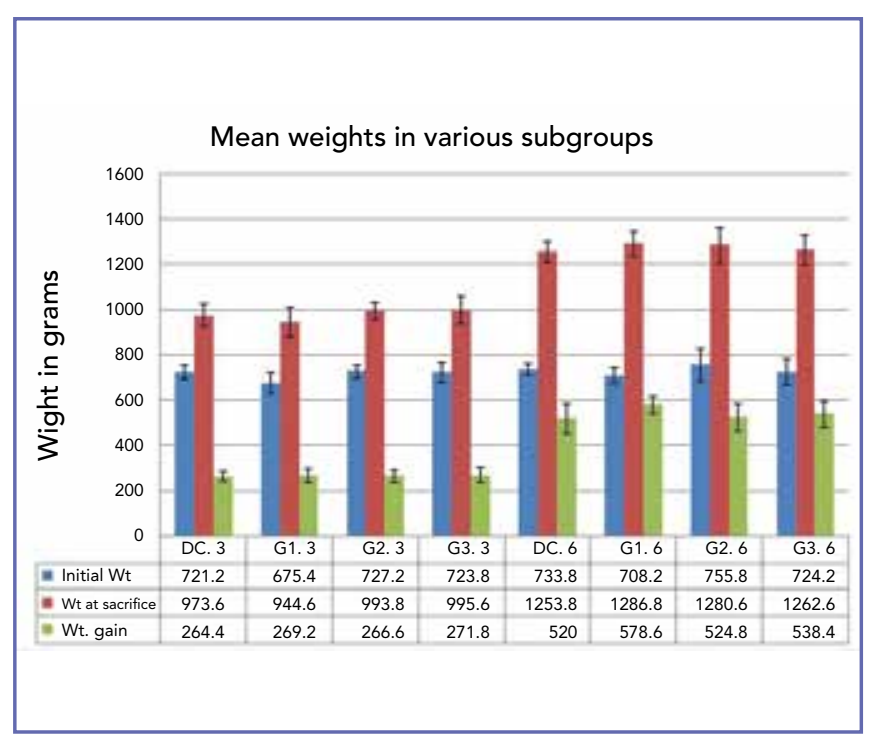

Figure 2. Graphical representation of initial weight, weight at sacrifice and weight gain for all the subgroups. There was no significant difference among the groups for any of the parameters at 3 or 6 months. ( Standard deviation for initial weight DC.3-29.719, G1.3- 46.420, G2.3- 29.115, G3.343.234 DC.6- 24.356, G1.6- 34.215, G2.6-69.830 ,G3.6.55 .006 49.719. Standard deviation for final weight-DC.349.71G1.3-66.42, G2.3-39.115, G3.3-63.234, DC.6-44.356, G1.6-54.215, G2.6-81.83, G3.6- 65.006 S.D. for weight gain DC.3- 22.952, G1.3- 32.322, G2.3-27.162,G3.3-35.088 DC.663.887, G1.6- 39.310, G2.6-61.227 , G3.6-55.089).

Table III. Table representing p values for comparisons for initial weight, weight at sacrifice and weight gain, mean synovial scores, mean total articular cartilage scores and mean COMP concentrations in the INTERVENTION arm of various groups. (Significant $\mathrm{p}$ values have been marked in bold font and with an asterisk $\left({ }^{*}\right)$ ).

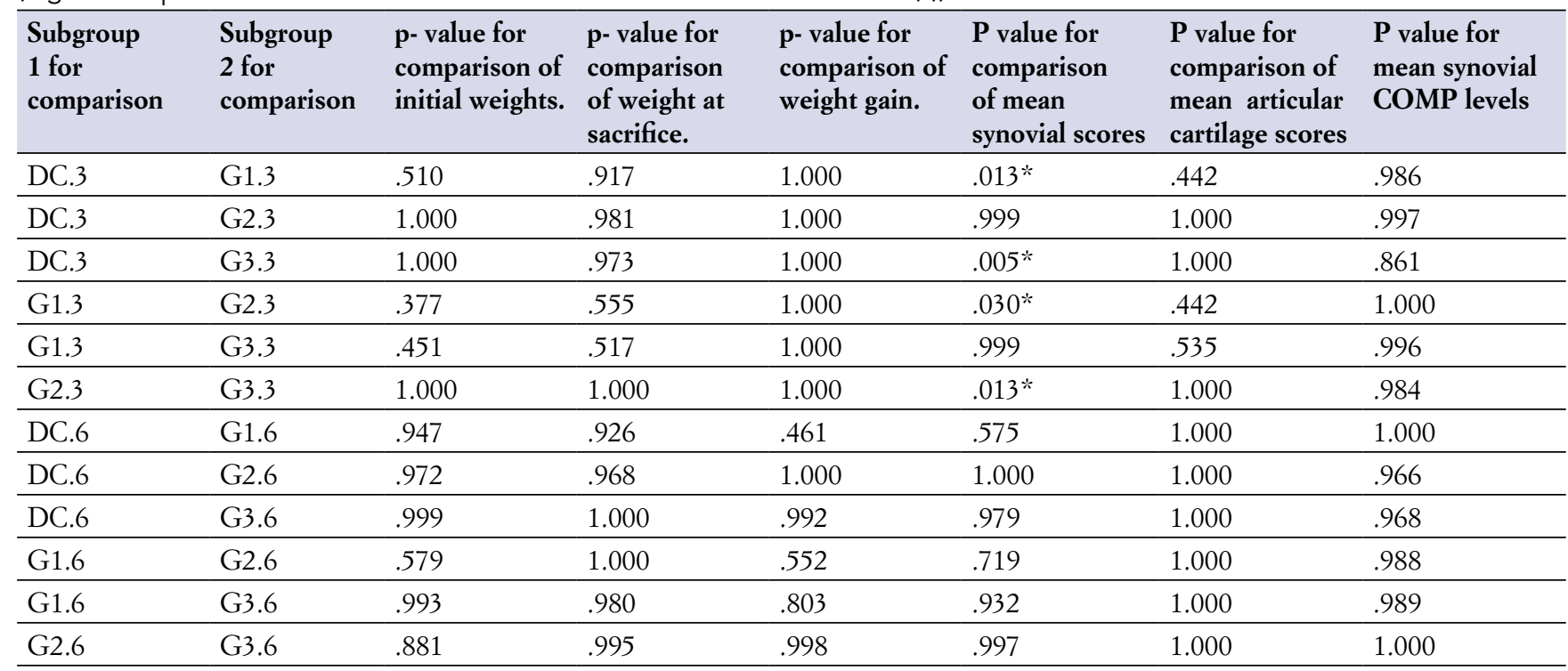




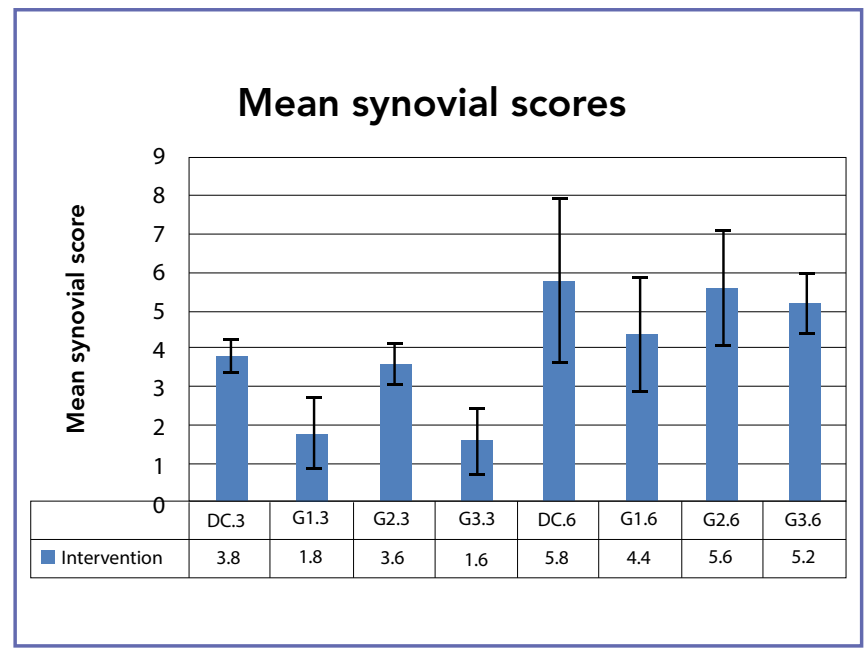

Figure 3. Graph representing the mean total synovial scores of intervention subgroup of various groups at 3 and 6 months. The scores were significantly better for the $\mathrm{G} 1.3$ and $\mathrm{G} 3.3$ compared to DC.3 and 2.3. ( Standard deviation for intervention Knee DC.3-.447, G1.3- 0.967, G2.3- .548, G3.3-.894, DC.6-2.168, G1.6-1.517, G2.6-1.517, G3.6-.837).

the PRP group and chitosan-PRP group were significantly better than the disease control group ( $\mathrm{p}$ value $=0.013$ $\& 0.005$ respectively) and chitosan gel only group ( $\mathrm{p}$ value $=0.03 \& 0.013$ ). However, there was no significant difference between the PRP and chitosan-PRP group in terms of mean synovitis score. There was no significant difference in the mean synovitis scores between all the subgroups at 6 months (table III). It was noted that although single injection of PRP and Chitosan-PRP resulted in better mean total synovial scores than Chitosan gel and disease control groups at 3 months, this effect was not sustained in the long term (6 months). It also appears that although PRP exerts an anti-inflammatory effect resulting in a decrease in synovial inflammation, the addition of chitosan gel did not improve its efficacy.

The mean total articular cartilage scores of the intervention arm are represented in figure 4 . There was no significant difference among mean total articular scores of intervention subgroups of all groups at 3 months and 6 months. This implies that single injection of PRP and chitosan-PRP does not seem to offer any significant protective effect on the cartilage. The mean synovial COMP concentrations for the intervention sub arms are represented in figure 5. There was no significant difference among mean COMP concentrations of intervention subgroups at 3 months (DC.3, G1.3, G2.3 and G3.3). Similarly, at 6 months, there was no significant difference among mean COMP concentrations of interven-

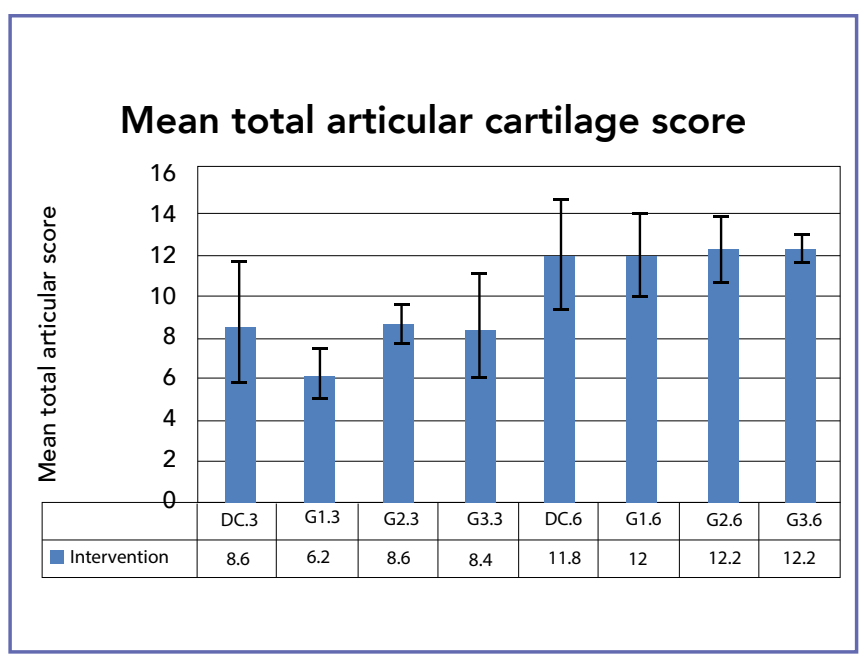

Figure 4. Graph representing mean total articular scores of intervention subgroups of various groups. No significant difference was noted among the groups at 3 or 6 months. (Standard deviation for intervention Knee DC.3- 3.050, G1.31.304, G2.3-.894,G3.3-2.608 DC.6- 2.864, G1.6-2.000, G2.6$1.643, \mathrm{G} 3.6 .-837)$.

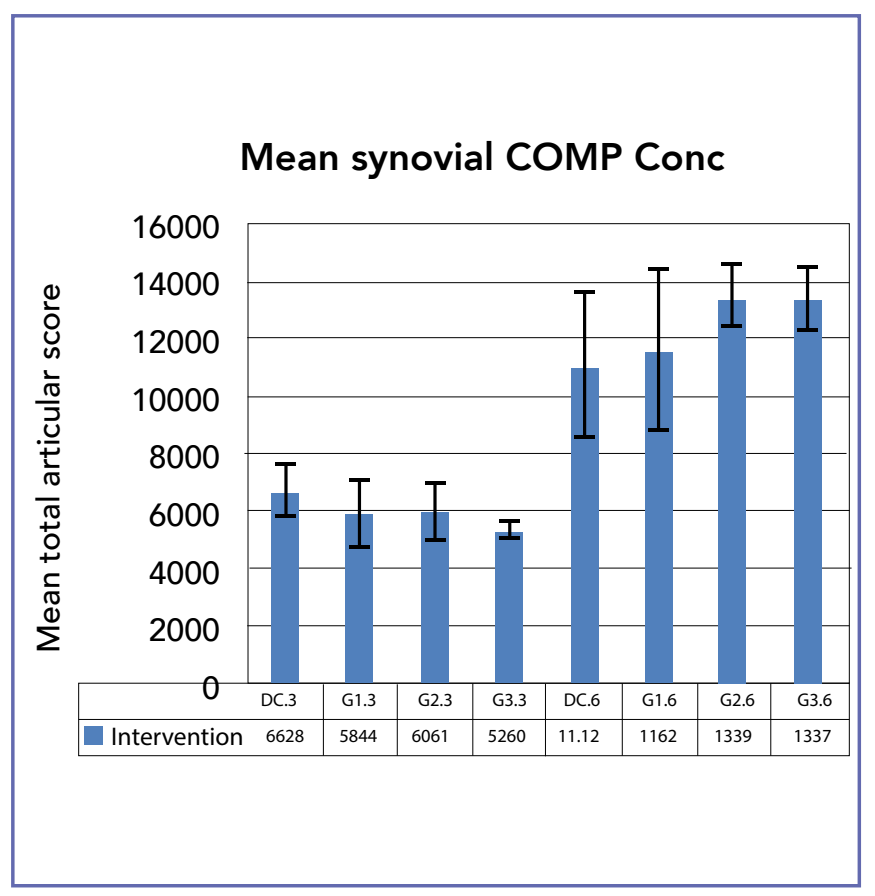

Figure 5. Graph representing mean COMP concentration of intervention subgroups of various groups. No significant difference was noted among the groups at 3 or 6 months (Standard deviation for intervention Knee DC.3- 1028.552, G1.3-1215.743， G2.3-872.709， G3.3-357.814， DC.62658.968, G1.6-2925.881, G2.6-1345.657, G3.6.- 1239.349). 
tion arms (DC.6, G1.6, G2.6 and G3.6). Images of synovi$\mathrm{al}$ and articular cartilage histological morphology are represented in figure $\mathbf{6}$ and figure $\mathbf{7}$ respectively.

No adverse events were noted in any of the groups.

\section{DISCUSSION}

As our understanding of the time limited efficacy of PRP evolved, attempts were made to use biomaterials with PRP, to see if the effect could be prolonged; this was started almost a decade ago. Since the initial work, there have been a variety of biomaterials which have been used along with PRP. Biomaterials like gelatin hydrogels (4), Chitosan (5), PLGA (poly lactic-co-glycolic acid) mesh and $\beta$-tricalcium phosphate scaffolds $(6,7)$ have been added to try and prolong and sustain the release of growth factors, with some success in specific scenarios.

Saito et al. (4) were the first to use biomaterials with PRP for intraarticular application in OA knee. They demonstrated the effects of PRP with gelatin biospheres in a surgically induced model of rabbit OA (ACL transection). 2 injections were given at an interval of 3 weeks. They evaluated the expression of proteoglycan core protein $\mathrm{mRNA}$ in the cartilage along with cartilage histology as a measure of outcome analysis. This study showed improved cartilage histology (as seen by 2 independent observers) and increased proteoglycan mRNA expression in the cartilage treated by PRP as compared to controls at 10 weeks.

Chitosan and PRP combinations have been studied in many in vitro and in vivo studies and is proven to be safe and nontoxic $(11,12)$. It has also been shown to enhance platelet aggregation, adhesion and increase glycoprotein III expression (10). Chitosan scaffolds with PRP have been shown to demonstrate a controlled and sustained release of the growth factors Transforming growth factor- $\beta 1$ and platelet derived growth factor- $\mathrm{AB}$ compared to PRP alone $(10,11)$ and also cause significant growth of mesenchymal stem cells (13). Chitosan and PRP combination has also found application in wound healing (14), filling of bone defects (15) and treatment of cartilage defects $(16,17)$. Mohmadi et al. (14) showed that Chitosan film combined with PRP resulted in better wound healing than PRP or Chitosan alone, in iatrogenically created wounds in rat model. Bi et al. (15) used a composite scaffold made of PRP, Chitosan and tri-calcium phosphate and showed that this composite was better in healing bone defects in tibia of goat than control groups at 16 weeks.

Chitosan based products have been widely used in cartilage related pathologies. A chitosan-based scaffold "BST-Car$\mathrm{Gel}^{\circledR}$ " in a randomized control trail has been shown to shown to be superior to microfractures alone for repair of

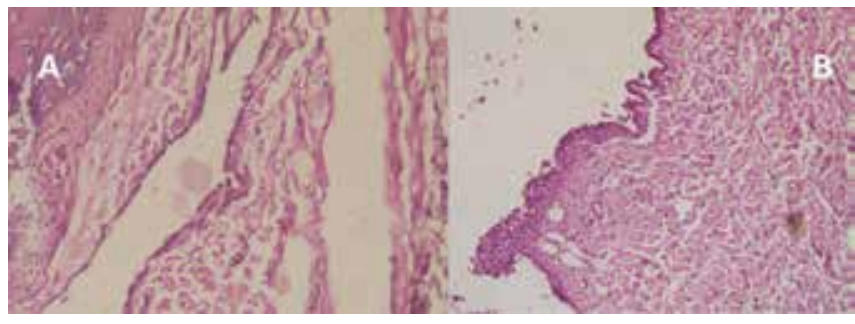

Figure 6. Hematoxylin and eosin stain showing synovial morphology. A. PRP treated knee (G1.3) showing no synovial hyperplasia (white arrow) and minimal inflammation at 3 months. B. Control (DC.3) knee showing 3 to 5 layered synovial hyperplasia (white arrow) and marked inflammation at 3 months.

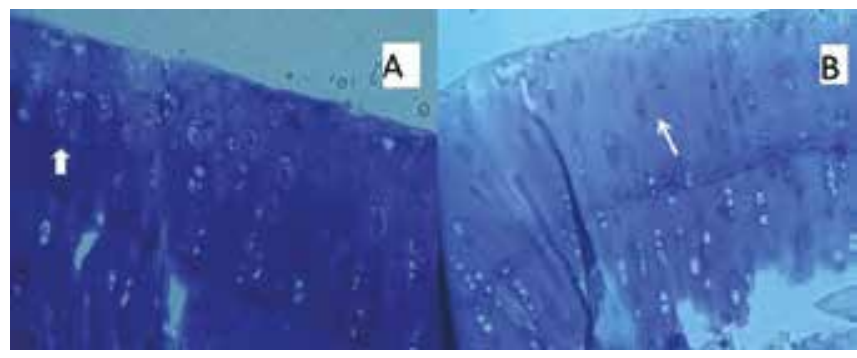

Figure 7. Toluidine Blue stain used for articular cartilage morphology. A. Chitosan PRP (G3.3) treated knee with a single superficial fissure, minimal loss of proteoglycan, normal cellularity (white arrow) and no duplication of tidemark. B. Knee from disease control group (DC.3) showing multiple superficial fissures, loss of proteoglycan, decreased cellularity (white arrow) and duplication of tidemark.

cartilage defects. Patients treated with chitosan scaffolds showed significantly greater filling of lesion and better repair tissue than cases with micro fractures (30). The Chitosan and PRP combinations have also been encouraging in cartilage defects. Segundo et al. (16) in their study on 12 rabbits compared a combination of PRP, chitosan and hydroxyapatite with no treatment in surgically created cartilage defects. They noted that significantly greater amount of tissue at the bone cartilage interface in the test group. In another study, Dwivedi et al. (17) surgically created chronic defects in cartilage of 8 rabbits; these defects were treated by bone marrow stimulation (BMS) with PRP or freezedried Chitosan with PRP. They noted that animals treated with BMS + Chitosan PRP had better articular scores at 8 weeks compared to BMS + PRP. They hypothesized that Chitosan/PRP implants reside in vivo for several weeks and have significant bioactivity, in contrast to PRP implants alone that are quickly degraded in one day. 
Though PRP has been used extensively for intraarticular use in osteoarthritis, there are no studies studying the effect of PRP in combination with Chitosan in a knee osteoarthritis model. In an attempt to replicate the positive effect of chitosan/PRP combination in other situations, we prepared an injectable combination of chitosan poloxamer gel with $\mathrm{PRP}$, to see whether the positive effects seen in cartilage defect studies could be translated to knee osteoarthritis.

The Dunkin Hartley guinea pigs develop weight induced knee osteoarthritis. The progression of knee osteoarthritis in this model has been proven to be co-relating to the weight gain of these animals $(20,23,31)$. We chose this model, as it is a naturally occurring model and its progression is proportionate and similar to humans $(29,31)$. The progression in surgically induced models is more rapid and in line with post traumatic arthritis than natural osteoarthritis. Moreover, the joint pathology in guinea pigs is affected by similar risk factors like age, weight gain and sedentary lifestyle $(29,31)$. For uniformity of preparation we have used allogenic PRP prepared from guinea pig blood. Multiple studies have supported the use of allogeneic PRP in animals and have shown it to be safe, reliable and more consistent with respect to concentration of platelets $(32,33)$. The PRP prepared from guinea pig blood for injection in PRP group had an absolute platelet count of $1,500,000 / \mu l$. We prepared a double concentrate PRP for Chitosan PRP group and the platelet count was approximately 7 times the baseline. Due to dilution from Chitosan poloxamer gel in the ratio 3:1(Chitosan: PRP) and absolute platelet count was 1,090,625/ $\mu$ l. These values of platelet concentrations were within at least 2 to 3 times the baseline counts for guinea pigs (24).

Injectable thermosensitive Chitosan poloxamer gel has been used as a carrier of drugs in various fields. The gel offers the advantage of improved bioavailability, sustained and prolonged drug release (34). Chitosan poloxamer gels have also been established as a drug delivery option to cartilage, and have been proven to be safe and excellent in this role $(35,36)$. There have been no studies combining Chitosan poloxamer gels with PRP have been reported in the literature. The gel used in our study had a sol to gel transition temperature of $30-32{ }^{\circ} \mathrm{C}$; it thus remained liquid at room temperature and turned to gel at body temperature. The gelling properties of Chitosan poloxamer gel remained intact after sterilization and even after dilution with PRP. The sol properties of gel at room temperature offer the advantage of easy injectability in joints of small animal like guinea pig with a 26 gauge needle. This is in contrast to Chitosan PRP scaffold preparations used by some of the previous authors where preparations had to be directly applied to cartilage or bone defects and could not be used as an injection $(16,37)$. The preparation by Diwidei et al. was injectable, but needed an 18 gauge needle, which may not be appropriate for small animals (17).

The synovitis scores were better in both treatments groups (PRP alone and Chitosan/PRP) than other groups (Disease control and Chitosan alone) at 3 months; no significant difference could be demonstrated between these. One issue could be the lower concentration of platelets in the Chitosan PRP group (due to dilution from Chitosan gel) and loss of intrinsic properties of Chitosan due to sterilization (26). Nevertheless, the gelling properties and Newtonian behavior of gel remained intact after sterilization of gel and addition of PRP. Although in our study, Chitosan poloxamer gel was not shown to enhance the anti-inflammatory effects of PRP, further studies are needed in different scenarios, with larger animal models to prove or disprove this point.

Mean articular cartilage score was measured by Mankin scoring and an increase in scores would have signified chondroprotective role of the treatment group. In the current study there was no significant difference between treatment and control groups in terms of mean articular cartilage score. It this may be due to the fact that we have collated our data at later periods in time ( 3 and 6 months) as compared to previous studies (maximum of 8 to 9 weeks), and the effect of PRP is known to wear out $(16,17)$.

We utilized synovial COMP level measurement as it provides an objective measure of the cartilage degradation and has been shown to correlate with the grade of $\mathrm{OA}$ in guinea pigs and humans $(38,39)$. Results of synovial fluid COMP levels were similar to that of mean articular cartilage score where in there was no significant difference in the COMP concentrations at 3 and 6 months between any of the groups.

We could not establish a beneficial role of Chitosan + PRP combination over PRP alone, and this may be due to the following limitations 1) lower concentration of platelets in the Chitosan PRP group (due to dilution from Chitosan gel); 2) inability to quantify any loss of intrinsic properties of Chitosan poloxamer gel due to sterilization (26); 3) inability to compensate lower concentration of platelets with more volume of Chitosan PRP due to small knee joints of the animal model used. A larger animal model may be more appropriate for such a study; 4) we were not able to shed light on the degradation profile of the Chitosan poloxamer gel preparation in vivo and in vitro. The degradation profile of our preparation may be different from freeze dried Chitosan glycerol phosphate preparation along with BMS used in some other studies (17).

\section{CONCLUSIONS}

Addition of chitosan poloxamer gel did not offer any additional advantage in prolonging efficacy of PRP in a Guin- 
ea pig Knee OA model. Nevertheless, the efficacy of single dose PRP, with or without Chitosan, has been again demonstrated in the short term, due to decreased synovitis scores.

\section{ACKNOWLEDGEMENTS}

This study was funded by internal institutional funds via a research grant. Indian Council of Medical Research (ICMR) thesis research grant was also awarded for this project.

\section{AUTHOR CONTRIBUTIONS}

TB was involved in the concept, design of study, performing the experiments, data collection and analysis. MSD was the senior guide and involved in study design, interpreting the result and editing the manuscript. SP gave the concept behind the study, the study design and edited the manuscript. DC was involved in the study design and edited manuscript. $\mathrm{AB}$ was involved in histological analysis and PRP preparation. BM was involved in performing the experiment, preparation of chitosan PRP and interpreting biomarker results. NK was involved in histological analysis.

\section{CONFLICT OF INTERESTS}

The authors declare that they have no conflicts of interests.

\section{REFERENCES}

1. Patel S, Dhillon MS, Aggarwal S, Marwaha N, Jain A. Treatment with platelet-rich plasma is more effective than placebo for knee osteoarthritis: a prospective, double-blind, randomized trial. Am J Sports Med 2013;41(2):356-64.

2. Kon E, Buda R, Filardo G, et al. Platelet-rich plasma: intra-articular knee injections produced favorable results on degenerative cartilage lesions. Knee Surg Sports Traumatol Arthrosc 2010;18(4):472-9.

3. Filardo G, Kon E, Buda R et al. Platelet-rich plasma intra-articular knee injections for the treatment of degenerative cartilage lesions and osteoarthritis. Knee Surg Sports Traumatol Arthrosc 2011;19(4):528-35.

4. Saito M, Takahashi KA, Arai E et al. Intra-articular administration of platelet-rich plasma with biodegradable gelatin hydrogel microspheres prevents osteoarthritis progression in the rabbit knee. Clin. Exp. Rheumatol 2009;27(2):201.

5. Busilacchi A, Gigante A, Mattioli-Belmonte M, et al. Chitosan stabilizes platelet growth factors and modulates stem cell differentiation toward tissue regeneration. Carbohydr Poly 2013;98(1):665-76.

6. Kurita J, Miyamoto M, Ishii Y et al. Enhanced vascularization by controlled release of platelet-rich plasma impregnated in biodegradable gelatin hydrogel. Ann Thorac Surg 2011;92(3):837-44.
7. Lee JH, Nam J, Kim HJ, Yoo JJ. Comparison of three different methods for effective introduction of platelet-rich plasma on PLGA woven mesh. Biomed Mater 2015;10(2):025002.

8. La WG, Yang HS. Heparin-Conjugated Poly (Lactic-Co-Glycolic Acid) Nanospheres Enhance Large-Wound Healing by Delivering Growth Factors in Platelet-Rich Plasma. Artif Organs 2015;39(4):388-94.

9. Sawamura K, Ikeda T, Nagae M et al. Characterization of in vivo effects of platelet-rich plasma and biodegradable gelatin hydrogel microspheres on degenerated intervertebral discs. Tissue Eng. Part A 2009;15(12):3719-27.

10. Deprés-Tremblay G, Chevrier A, Tran-Khanh N, et al. Chitosan inhibits platelet-mediated clot retraction, increases platelet-derived growth factor release, and increases residence time and bioactivity of platelet-rich plasma in vivo. Biomed Mater 2017 10;13(1):015005.

11. Shen EC, Chou TC, Gau CH, Tu HP, Chen YT, Fu E. Releasing growth factors from activated human platelets after chitosan stimulation: a possible bio-material for platelet-rich plasma preparation. Clin. Oral Implants Res 2006;17(5):572-8.

12. Shimojo AA, Perez AG, Galdames SE, et al. Stabilization of porous chitosan improves the performance of its association with platelet-rich plasma as a composite scaffold. Mater. Sci Eng C 2016;60:538-46.

13. Shimojo AA, Galdames SE, Perez AG, et al. In vitro performance of injectable chitosan-tripolyphosphate scaffolds combined with platelet-rich plasma. J Tissue Eng Regen Med 2016;13(1):21-30

14. Mohammadi R, Mehrtash M, Mehrtash M, et al. Effect of platelet rich plasma combined with chitosan biodegradable film on full-thickness wound healing in rat model. Bulletin of Emergency \& Trauma 2016;4(1):29.

15. Bi L, Cheng W, Fan H, Pei G. Reconstruction of goat tibial defects using an injectable tricalcium phosphate/chitosan in combination with autologous platelet-rich plasma. Biomaterials 2010;31(12):3201-11.

16. Segundo FA, Costa EI, Azevedo AS et al. Platelet-rich plasma, hydroxyapatite, and chitosan in the bone and cartilaginous regeneration of femoral trochlea in rabbits: clinical, radiographic, and histomorphometric evaluations. J. Healthc Eng 2018;2018.

17. Dwivedi G, Chevrier A, Hoemann CD, Buschmann MD Injectable freeze-dried chitosan-platelet-rich-plasma implants improve marrow-stimulated cartilage repair in a chronic-defect rabbit model. J Tissue Eng Regen Med 2019;13(4):599-611.

18. Chen $\mathrm{CH}$, Chen SH, Mao SH et al. Injectable thermosensitive hydrogel containing hyaluronic acid and chitosan as a barrier for prevention of postoperative peritoneal adhesion. Carbohydr Polym 2017;173:721-31.

19. Deng Y, Ren J, Chen G, et al. Injectable in situ cross-linking chitosan-hyaluronic acid based hydrogels for abdominal tissue regeneration. Sci Rep 2017;7(1):1-3.

20. Chouhan DK, Dhillon MS, Patel S, Bansal T, et al. Multiple platelet-rich plasma injections versus single platelet-rich plasma injection in early osteoarthritis of the knee: an experimental study in a guinea pig model of early knee osteoarthritis. Am J Sports Med 2019;47(10):2300-7.

21. Padulo J, Oliva F, Frizziero A, Maffulli N. Basic principles and recommendations in clinical and field Science Research: 2018 update. MLTJ 2018; 8(3): $305-307$. 
22. Nagata MJ, Messora MR, Furlaneto FA, et al. Effectiveness of two methods for preparation of autologous platelet-rich plasma: an experimental study in rabbits. Eur J Dent 2010;4(04):395-402.

23. Kanwat H, Singh DM, Kumar CD, et al. The effect of intra-articular allogenic platelet rich plasma in Dunkin-Hartley guinea pig model of knee osteoarthritis. MLTJ 2017;7(3):426.

24. Wagner JE. The biology of the guinea pig. Academic Press 2014.

25. Cho HH, Jang S, Lee SC, et al. Effect of neural-induced mesenchymal stem cells and platelet-rich plasma on facial nerve regeneration in an acute nerve injury model. Laryngoscope 2010;120(5):907-13.

26. Kashikar VS, Gonjari ID. In situ gelling systems of ofloxacin: Comparative performance of in vivo precorneal drainage and pharmacokinetic study. Asian J Pharm 2014;7(1).

27. Wei L, Fleming BC, Sun X, et al. Comparison of differential biomarkers of osteoarthritis with and without posttraumatic injury in the Hartley guinea pig model. J Orthop Res 2010;28(7):900-6.

28. Underwood W, Anthony R, Cartner S, et al. AVMA guidelines for the euthanasia of animals: 2013 edition. Schaumburg, IL: American Veterinary Medical Association.

29. Kraus VB, Huebner JL, DeGroot J, Bendele A. The OARSI histopathology initiative-recommendations for histological assessments of osteoarthritis in the guinea pig. Osteoarthr Cartil 2010;18:S35-52.

30. Shive MS, Stanish WD, McCormack R, et al. BST-CarGel ${ }^{\circledR}$ treatment maintains cartilage repair superiority over microfracture at 5 years in a multicenter randomized controlled trial. Cartilage 2015;6(2):62-72.
31. Bendele AM. Animal models of osteoarthritis. J Musculoskelet Neuronal Interact 2001;1(4):363-76.

32. Kasten P, Vogel J, Geiger F, Niemeyer P, et al. The effect of platelet-rich plasma on healing in critical-size long-bone defects. Biomaterials 2008;29(29):3983-92.

33. Zhang ZY, Huang AW, Fan JJ, et al. The potential use of allogeneic platelet-rich plasma for large bone defect treatment: immunogenicity and defect healing efficacy. Cell Transplant 2013;22(1):175-87.

34. Khan S, Warade S, Singhavi DJ. Improvement in ocular bioavailability and prolonged delivery of tobramycin sulfate following topical ophthalmic administration of drug-loaded mucoadhesive microparticles incorporated in thermosensitive in situ gel. J Ocul Pharmacol Ther 2018;34(3):287-97.

35. Park KM, Lee SY, Joung YK, et al. Thermosensitive chitosanPluronic hydrogel as an injectable cell delivery carrier for cartilage regeneration. Acta Biomater 2009;5(6):1956-65.

36. Kang ML, Kim JE, Im GI. Thermoresponsive nanospheres with independent dual drug release profiles for the treatment of osteoarthritis. Acta Biomater 2016;39:65-78.

37. Oktay E, Demiralp B, Demiralp B, et al. Effects of platelet-rich plasma and chitosan combination on bone regeneration in experimental rabbit cranial defects. J Oral Implantol 2010;36(3):175-84.

38. Petersson IF, Boegard T, Dahlstrom J, et al. Bone scan and serum markers of bone and cartilage in patients with knee pain and osteoarthritis. Osteoarthr Cartil 1998;6:33-9.

39. Conrozier T, Saxne T, Fan CS, et al. Serum concentrations of cartilage oligomeric matrix protein and bone sialoprotein in hip osteoarthritis: A one year prospective study. Ann Rheum Dis 1998;57:527-32. 